Noname manuscript No.

(will be inserted by the editor)

\title{
Liberal approaches to ranking infinite utility streams: When can we avoid interference?
}

\author{
José Carlos R. Alcantud
}

Received: - / Accepted: -

\begin{abstract}
In this work we analyse social welfare relations on sets of finite and infinite utility streams that satisfy various types of liberal non-interference principles. Earlier contributions have established that (finitely) anonymous and strongly Paretian quasiorderings exist that verify non-interference axioms together with weak preference continuity and further consistency. Nevertheless Mariotti and Veneziani [16] prove that a fully liberal non-interfering view of a finite society leads to dictatorship if the weak Pareto principle is imposed. We first prove that this impossibility result vanishes when we extend the horizon to infinity. Then we investigate a related problem: namely, the possibility of combining "standard" semicontinuity with efficiency in the presence of noninterference. We provide several impossibility results that prove that there is a generalised incompatibility between relaxed forms of continuity and noninterference principles, both under ordinal and cardinal views of the problem.
\end{abstract}

José Carlos R. Alcantud

Campus Unamuno. Edificio FES.

E37007 Salamanca, Spain.

Personal webpage: http://web.usal.es/jcr

E-mail: jcr@usal.es Tel: +34-923-294640. Fax: +34-923-294686. 


\section{Introduction}

In relation with the analysis of criteria for comparing allocations to a finite society, Hammond's [10] characterization of the leximin ordering is based on anonymity, the strong Pareto axiom, and a principle now called Hammond Equity. It has been recently proven that in the presence of anonymity and the strong Pareto principle, Hammond Equity is equivalent to a liberal noninterference property called Harm Principle, henceforth HP (cf., Mariotti and Veneziani [14]). The generic idea of liberal non-interference is that an individual has the right to make society remain passive in all circumstances of change in her welfare, provided that the welfare of no other individual is affected. In particular, when 'change' means only damage the Harm Principle is obtained, and when it means only benefit a 'dual' property called the Individual Benefit Principle, henceforth IBP, comes forward. Thus as the authors acknowledge, the alternative characterization of the leximin social ranking in [14] seems fairly surprising since HP does not embody any egalitarian consideration while Hammond Equity is a strongly egalitarian property. In related lines of work, extensions of the analysis to the case of the leximax criterion and also to the case of infinitely-lived societies appear in Mariotti and Veneziani $[16]$ and Lombardi and Veneziani [12,13]. They appeal to the finite- or infinitedimensional versions of either HP (leximin) or IBP (leximax). In particular, preference continuities permit to characterize infinite extensions of the leximin criterion both on the basis of Hammond Equity (cf., Asheim and Tungodden [4]) and of adapted versions of HP. Nevertheless, [13] shows that in the evaluation of infinitely long streams by orderings, anonymity, the strong Pareto axiom, and preference continuity properties are incompatible with full noninterference - understood as the combination of the spirits of HP and IBP. Restricting ourselves to a finite economy, Mariotti and Veneziani [16] prove that a fully liberal non-interfering view of the society leads to dictatorship if the weak Pareto principle is imposed.

In this paper we first prove that the latter impossibility result vanishes when we extend the horizon to infinity (cf., Section 3). To be more precise, in the infinite setting non-dictatorship (resp., anonymity) is compatible with full non-interference even if we restrict ourselves to using monotonic social orderings with the weak Pareto property (resp., a restricted version of the weak Pareto property) and some minimal equity requirement. Afterwards we explore the consequences of imposing weak standard continuity properties to non-interfering criteria (cf., Section 4). For the case of a finite society, it is implicitly known that topological continuity is incompatible with equity and efficiency in the presence of liberal non-interference. A very direct coarse argument derives from the fact that the leximin (resp., leximax) criterion for comparing allocations in a finite society lacks upper (resp., lower) semicontinuity with respect to the sup topology. From this it is trivial that no social ordering can verify anonymity, the strong Pareto axiom, and HP (resp., IBP) under upper (resp., lower) semicontinuity with respect to the sup topology. We provide more accurate reasons for the disclosed conflict by proving that 
anonymity and transitivity play no role in the aforementioned incompatibilities, and also that impossibility remains when an extremely mild technical condition replaces strong Pareto instead. Turning now to the infinite case, the situation is similar. In particular we prove that semicontinuity imposes bounds even on non-interfering criteria that satisfy neither transitivity nor the full strength of the completeness axiom, for they must violate a very relaxed version of the Pareto postulate that only affects comparisons between eventually constant and coincident streams. Renouncing completeness in this result is not a merely formal move since many of the social welfare relations that are preeminent in the intergenerational analyses only verify the restricted version of completeness that we use. Then we elaborate on less demanding views of non-interference that scarcely provide some routes of escape to the generalised impossibilities that arise. We end up our paper with some conclusions.

\section{Notation and axioms}

A social welfare relation (SWR) is a binary relation $\succcurlyeq$ on $\mathbf{X}=[0,1]^{n}$ with $n \in \mathbb{N} \cup\{\infty\}$. When $n \in \mathbb{N}$ it is interpreted as the cardinality of a finite set of agents. When $n=\infty$, we interpret that there is a countably infinite set of agents $\mathbb{N}$ or of generations, and $\mathbf{X}$ represents a domain of bounded utility sequences or infinite-horizon utility streams. Unless we state otherwise, it is assumed that $\succcurlyeq$ is reflexive. Its asymmetric factor is denoted by $\succ$ (i.e., $\mathbf{x} \succ \mathbf{y}$ iff $\mathbf{x} \succcurlyeq \mathbf{y}$ but not $\mathbf{y} \succcurlyeq \mathbf{x}$ ), and its symmetric factor is denoted by $\sim$ (i.e., $\mathbf{x} \sim \mathbf{y}$ iff $\mathbf{x} \succcurlyeq \mathbf{y}$ and $\mathbf{y} \succcurlyeq \mathbf{x}$ ). If $\succcurlyeq$ is an ordering (i.e., complete and transitive) then we call it a social welfare ordering or SWO.

We develop the notation for the case when $\mathbf{X}=[0,1]^{\mathbb{N}}$. Its elements $\mathbf{x}=\left(x_{1}, \ldots, x_{n}, \ldots \ldots.\right) \in \mathbf{X}$ list the allocations to agents/generations if $\mathbf{x}$ is chosen. By $(y)_{c o n}$ we mean the constant sequence $(y, y, \ldots)$, and similarly $\left(x_{1}, \ldots, x_{k},(y)_{\text {con }}\right)=\left(x_{1}, \ldots, x_{k}, y, y, \ldots\right)$ denotes an eventually constant sequence. Denote by ${ }_{1} \mathbf{x}_{T}=\left(x_{1}, \ldots, x_{T}\right)$ and ${ }_{T+1} \mathbf{x}=\left(x_{T+1}, x_{T+2}, \ldots\right)$ the $T$ head and the $T$-tail of $\mathbf{x} \in \mathbf{X}$, thus $\mathbf{x}={ }_{1} \mathbf{x}=\left({ }_{1} \mathbf{x}_{n},{ }_{n+1} \mathbf{x}\right)$ for each $n \in \mathbb{N}$. When the intergenerational terminology is adopted, the first component or generation is often called the present. We say that the streams $\mathbf{x}, \mathbf{y} \in \mathbf{X}$ are eventually coincident if there is $j \in \mathbb{N}$ such that $x_{i}=y_{i}$ for each $i>j$.

We write $\mathbf{x} \geqslant \mathbf{y}$ if $x_{i} \geqslant y_{i}$ for each $i=1,2, \ldots$, and $\mathbf{x} \gg \mathbf{y}$ if $x_{i}>y_{i}$ for each $i=1,2, \ldots$. Also, $\mathbf{x}>\mathbf{y}$ means $\mathbf{x} \geqslant \mathbf{y}$ and $\mathbf{x} \neq \mathbf{y}$.

We are concerned with axioms of different nature for SWRs. We state them for $\mathbf{X}=[0,1]^{\mathbb{N}}$ but they can be easily regarded as axioms on $\mathbf{X}=[0,1]^{n}$ with $n \in \mathbb{N}$ too, as is dutifully clarified along the exposition when needed.

Firstly we introduce equity axioms of two different classes for a SWR on $\mathbf{X}=[0,1]^{\mathbb{N}}$. Anonymity is the usual "equal treatment of all generations" postulate à-la-Sidgwick and Diamond.

Axiom AN (Anonymity). For all $\mathbf{x}, \mathbf{y} \in \mathbf{X}$, if there exist $i, j \in \mathbb{N}$ such that $x_{i}=y_{j}$ and $x_{j}=y_{i}$, and for $k \in \mathbb{N}-\{i, j\}, x_{k}=y_{k}$, then $\mathbf{x} \sim \mathbf{y}$. 
We now recall a consequentialist equity axiom that implements preference for egalitarian allocations of utilities among generations. Axiom HE below states that in case of a conflict between two generations, every other generation being as well off, the stream where the least favoured generation is better off must be weakly preferred.

Axiom HE (Hammond Equity). If $\mathbf{x}, \mathbf{y} \in \mathbf{X}$ are such that $x_{j}>y_{j}>y_{k}>$ $x_{k}$ for some $j, k \in \mathbb{N}$, and $x_{t}=y_{t}$ when $j \neq t \neq k$, then $\mathbf{y} \succcurlyeq \mathbf{x}$.

Further we are concerned with the following axiom that was introduced in Asheim and Tungodden [5].

Axiom HEF (Hammond Equity for the Future). If $\mathbf{x}, \mathbf{y} \in \mathbf{X}$ are such that $\mathbf{x}=\left(x_{1},(x)_{c o n}\right), \mathbf{y}=\left(y_{1},(y)_{c o n}\right)$ and $x_{1}>y_{1}>y>x$, then $\mathbf{y} \succcurlyeq \mathbf{x}$.

HEF states the following ethical restriction on the ranking of streams where the level of utility is constant from the second period on and the present generation is better-off than the future: If the sacrifice by the present conveys a higher utility for all future generations, then such trade off is weakly preferred.

In a different vein, Mariotti and Veneziani [16,14] introduce non-interference conditions in the context of a finite society. Under additional requirements they are intimately related to HE (cf., Mariotti and Veneziani [14, p. 127]). We proceed to recall their infinite counterparts, which are extensively analyzed in Lombardi and Veneziani $[12,13]$. Their respective versions for finite-length streams are the same except in that the restriction of the thesis to eventually coincident vectors does not apply.

Axiom HP (Harm Principle). Suppose $\mathbf{x}, \mathbf{y} \in \mathbf{X}$ are eventually coincident and $\mathbf{x} \succ \mathbf{y}$. Consider two streams $\mathbf{x}^{\prime}, \mathbf{y}^{\prime} \in \mathbf{X}$ such that: for some $i \in \mathbb{N}, j \neq i$ implies $x_{j}^{\prime}=x_{j}$ and $y_{j}^{\prime}=y_{j}$. If $x_{i}^{\prime}<x_{i}$ and $y_{i}^{\prime}<y_{i}$ then $x_{i}^{\prime}>y_{i}^{\prime}$ implies $\mathbf{x}^{\prime} \succ \mathbf{y}^{\prime}$.

Axiom IBP (Individual Benefit Principle). Suppose $\mathbf{x}, \mathbf{y} \in \mathbf{X}$ are eventually coincident and $\mathbf{x} \succ \mathbf{y}$. Consider two streams $\mathbf{x}^{\prime}, \mathbf{y}^{\prime} \in \mathbf{X}$ such that: for some $i \in \mathbb{N}, j \neq i$ implies $x_{j}^{\prime}=x_{j}$ and $y_{j}^{\prime}=y_{j}$. If $x_{i}^{\prime}>x_{i}$ and $y_{i}^{\prime}>y_{i}$ then $x_{i}^{\prime}>y_{i}^{\prime}$ implies $\mathbf{x}^{\prime} \succ \mathbf{y}^{\prime}$.

Quoting from [16], the core of these non-interference principles is the following idea (we adopt the intergenerational terminology): changes in one generation's welfare that leave all other generations unaffected should not be a motive for penalising that generation in the social judgement, whether the change involves a damage (HP) or a benefit (IBP) for that generation. A penalisation means a switch against its interest in the society's strict ranking of the distributions (with respect to the ranking of the original distributions). This explanation adapts to the other usual interpretations in a direct manner.

Relaxed versions of HP and IBP where the conclusion of the axioms merely state $\mathbf{y}^{\prime} \nsucc \mathbf{x}^{\prime}$ instead of $\mathbf{x}^{\prime} \succ \mathbf{y}^{\prime}$, are respectively called Weak Harm Principle (WHP) and Weak Individual Benefit Principle (WIBP): v., Mariotti and Veneziani [16]. 
Social evaluations need to account for some kind of efficiency too. Various axioms capture the general principle that with respect to a given infinite utility stream, adequate changes must produce socially better streams if every generation is at least as well off after the change. The Weak Dominance axiom captures the following spirit: Improving the welfare of exactly one generation suffices to produce a socially better stream. In turn, the Weak Pareto axiom requests that all generations increase their utility in order to obtain a socially better stream. The Strong Pareto axiom imposes that if at least one generation increases its utility then the resulting stream is socially better thus Strong Pareto and Weak Dominance coincide over sets of finite-length vectors. Formally:

Axiom WD (Weak Dominance). If $\mathbf{x}, \mathbf{y} \in \mathbf{X}$ and there is $j \in \mathbb{N}$ such that $x_{j}>y_{j}$, and $x_{i}=y_{i}$ for all $i \neq j$, then $\mathbf{x} \succ \mathbf{y}$.

Axiom WP (Weak Pareto). If $\mathbf{x}, \mathbf{y} \in \mathbf{X}$ and $\mathbf{x} \gg \mathbf{y}$, then $\mathbf{x} \succ \mathbf{y}$.

Axiom SP (Strong Pareto). If $\mathbf{x}, \mathbf{y} \in \mathbf{X}$ and $\mathbf{x}>\mathbf{y}$ then $\mathbf{x} \succ \mathbf{y}$.

Another relaxed form of Strong Pareto that is unrelated both to WP and WD is the uncontroversial Monotonicity.

Axiom $\mathbf{M}$ (Monotonicity). If $\mathbf{x}, \mathbf{y} \in \mathbf{X}$ and $\mathbf{x}>\mathbf{y}$ then $\mathbf{x} \succcurlyeq \mathbf{y}$.

Observe that SWOs that verify M and WD verify SP too.

Finally, we list some semicontinuity properties for the context $\mathbf{X} \subseteq \mathbb{R}^{\mathbb{N}}$ and below we discuss how they adapt to the case $\mathbf{X} \subseteq \mathbb{R}^{n}, n \in \mathbb{N}$ :

Axiom RUSC (Restricted upper semicontinuity with respect to the sup topology). For each $\mathbf{x} \in \mathbf{X}$ eventually constant, $\{\mathbf{y} \in \mathbf{X}: \mathbf{y} \succcurlyeq \mathbf{x}\}$ is closed with respect to the sup topology.

Axiom RLSC (Restricted lower semicontinuity with respect to the sup topology). For each $\mathbf{x} \in \mathbf{X}$ eventually constant, $\{\mathbf{y} \in \mathbf{X}: \mathbf{x} \succcurlyeq \mathbf{y}\}$ is closed with respect to the sup topology.

In general, the sup topology is finer than the product topology but when $\mathbf{X} \subseteq \mathbb{R}^{n}$ with $n \in \mathbb{N}$, both topologies coincide with the Euclidean topology. Also in this context, RUSC/RLSC are the ordinary USC/LSC (upper/lower semicontinuity with respect to the sup topology). As usual, continuity means the conjunction of upper and lower semicontinuity.

\section{The possibility of non-interference with an infinite horizon}

In the context of SWOs on allocations to a finite society with two or more agents, there are linear rankings that verify SP, HP and IBP (e.g., lexicographic orders) but there are not SWOs that verify WP, WHP, WIBP, and AN because all SWOs that verify WP, WHP and WIBP are dictatorial by a generation (cf., Mariotti and Veneziani [16, Theorem 1]). For example, lexicographic orders are 
dictatorial by the first generation. Such impossibility result implies a simple Corollary for infinitely-lived societies that uses the following axiom:

Axiom RWD (Restricted Weak Dominance). If $\mathbf{x}, \mathbf{y} \in \mathbf{X}$ are eventually constant, there is $j \in \mathbb{N}$ such that $x_{j}>y_{j}$, and $x_{i}=y_{i}$ for all $i \neq j$, then $\mathbf{x} \succ \mathbf{y}$.

Corollary 1 There is no $S W O$ on $\boldsymbol{X}=[0,1]^{\mathbb{N}}$ that verifies $R W D$, WHP, $W I B P$, and $A N$.

Proof If $\succcurlyeq$ is a SWO on $\mathbf{X}=[0,1]^{\mathbb{N}}$ that verifies RWD, WHP, WIBP, and $\mathrm{AN}$, then the expression

$$
(x, y) R\left(x^{\prime}, y^{\prime}\right) \text { iff }\left(x, y, 0_{c o n}\right) \succcurlyeq\left(x^{\prime}, y^{\prime}, 0_{c o n}\right)
$$

produces a SWO on $[0,1]^{2}$ that verifies WP, WHP, WIBP, and AN. This is against Mariotti and Veneziani [16, Theorem 1].

We proceed to further explore the implications of non-interference postulates with an infinite horizon. We appeal to the following non-dictatorship axioms (cf., Chichilnisky [9], also Sakai [17,18], Asheim et al. [3]) that impose that the comparisons between pairs of streams do not depend on the welfare levels of present, resp., future, generations only.

Axiom NDP (Non-Dictatorship of the Present). The following is not true: If $\mathbf{x}, \mathbf{y} \in \mathbf{X}$ are such that $\mathbf{x} \succ \mathbf{y}$, there is $i \in \mathbb{N}$ for which $j \geqslant i$ and $\mathbf{z}, \mathbf{w} \in \mathbf{X}$ imply $\left({ }_{1} \mathbf{x}_{j},{ }_{j+1} \mathbf{z}\right) \succ\left({ }_{1} \mathbf{y}_{j},{ }_{j+1} \mathbf{w}\right)$.

Axiom NDF (Non-Dictatorship of the Future). The following is not true: If $\mathbf{x}, \mathbf{y} \in \mathbf{X}$ are such that $\mathbf{x} \succ \mathbf{y}$, there is $i \in \mathbb{N}$ for which $j \geqslant i$ and $\mathbf{z}, \mathbf{w} \in \mathbf{X}$ imply $\left({ }_{1} \mathbf{z}_{j},{ }_{j+1} \mathbf{x}\right) \succ\left({ }_{1} \mathbf{w}_{j},{ }_{j+1} \mathbf{y}\right)$.

Sakai [18, Propositions 1 and 2] provides the following simple relationships. Let $\succcurlyeq$ be a binary relation on $\mathbf{X}$. If $\succcurlyeq$ verifies AN and there are $\mathbf{x}, \mathbf{y} \in \mathbf{X}$ such that $\mathbf{x} \succ \mathbf{y}$ then $\succcurlyeq$ verifies NDP. If $\succcurlyeq$ verifies RWD then $\succcurlyeq$ verifies NDF.

In contrast with Corollary 1, Theorem 1 below indicates that the negative result in Mariotti and Veneziani [16] is overturned when moving to infinite societies, in the sense that the combination of WP with the strict versions of the non-interference principles does not result in a dictatorship.

Theorem 1 There are SWOs on $\boldsymbol{X}=[0,1]^{\mathbb{N}}$ that verify $M, W P, H E F, H P$, $I B P, N D P$, and $N D F$.

Proof We define the following binary relation $\succcurlyeq$ on $\mathbf{X}: \mathbf{x} \succcurlyeq \mathbf{y}$ if and only if either $\liminf n_{n}\left(x_{n}\right)>\liminf _{n}\left(y_{n}\right)$ or $\liminf _{n}\left(x_{n}\right)=\liminf _{n}\left(y_{n}\right)$ and $x_{1} \geqslant$ $\left.y_{1}\right)$. This is a lexicographic composition of a long-run criterion and dictatorship of the present. Thus it is routine to check that $\succcurlyeq$ is a complete preorder. Its asymmetric part is defined by: $\mathbf{x} \succ \mathbf{y}$ if and only if either $\liminf _{n}\left(x_{n}\right)>$ $\liminf \operatorname{in}_{n}\left(y_{n}\right)$ or $\operatorname{~} \liminf _{n}\left(x_{n}\right)=\liminf _{n}\left(y_{n}\right)$ and $\left.x_{1}>y_{1}\right)$. 
In order to prove $\mathrm{M}$, take $\mathbf{x}, \mathbf{y} \in \mathbf{X}$ with $\mathbf{x} \geqslant \mathbf{y}$ thus $\liminf \operatorname{in}_{n}\left(x_{n}\right) \geqslant$ $\liminf \operatorname{in}_{n}\left(y_{n}\right)$. If $\liminf \sin _{n}\left(x_{n}\right)>\liminf _{n}\left(y_{n}\right)$ we obtain $\mathbf{x} \succ \mathbf{y} \cdot \operatorname{If} \liminf { }_{n}\left(x_{n}\right)=$ $\liminf \operatorname{in}_{n}\left(y_{n}\right)$ then the fact that $x_{1} \geqslant y_{1}$ yields $\mathbf{x} \succcurlyeq \mathbf{y}$.

In order to prove WP, take $\mathbf{x}, \mathbf{y} \in \mathbf{X}$ with $\mathbf{x} \gg \mathbf{y}$ thus $\liminf { }_{n}\left(x_{n}\right) \geqslant$ $\lim \inf _{n}\left(y_{n}\right)$. We proceed as above to check $\mathbf{x} \succ \mathbf{y}$.

The proof that $\succcurlyeq$ verifies a reinforced version of HEF is direct: If $\mathbf{x}=$ $\left(x_{1},(x)_{\text {con }}\right), \mathbf{y}=\left(y_{1},(y)_{\text {con }}\right)$ and $x_{1}>y_{1}>y>x$ then $\mathbf{y} \succ \mathbf{x}$ because $\lim \inf _{n}\left(y_{n}\right)=y>x=\liminf _{n}\left(x_{n}\right)$.

Let us now prove HP. Suppose $\mathbf{x}, \mathbf{y} \in \mathbf{X}$ are eventually coincident with $\mathbf{x} \succ \mathbf{y}$, thus $\liminf \operatorname{in}_{n}\left(x_{n}\right)=\liminf _{n}\left(y_{n}\right)$ and $x_{1}>y_{1}$. Consider $\mathbf{x}^{\prime}, \mathbf{y}^{\prime} \in \mathbf{X}$ such that: for some $i \in \mathbb{N}, j \neq i$ implies $x_{j}^{\prime}=x_{j}$ and $y_{j}^{\prime}=y_{j}$. Since $\liminf \operatorname{in}_{n}\left(x_{n}^{\prime}\right)=$ $\liminf _{n}\left(x_{n}\right)$ and $\liminf _{n}\left(y_{n}^{\prime}\right)=\liminf _{n}\left(y_{n}\right)$, in case that $x_{i}^{\prime}<x_{i}, y_{i}^{\prime}<y_{i}$ and $y_{i}^{\prime}<x_{i}^{\prime}$ then we deduce $\mathbf{x}^{\prime} \succ \mathbf{y}^{\prime}$ irrespective of $i$ being 1 or greater.

The proof that $\succcurlyeq$ verifies IBP is analogous to the argument for HP.

Sakai [18, Proposition 1] yields NDP.

Finally, in order to check that there is no dictatorship of the future observe that if $\mathbf{x}=\left(1_{\text {con }}\right), \mathbf{y}=\left(0,1_{\text {con }}\right)$ it is true that $\mathbf{x} \succ \mathbf{y}$ but for each $i>1$ and $j \geqslant i$, if $\mathbf{z}=\left(0_{\text {con }}\right), \mathbf{w}=\left(1_{\text {con }}\right)$ one has $\left({ }_{1} \mathbf{w}_{j}, j+1 \mathbf{y}\right) \succ\left({ }_{1} \mathbf{z}_{j},{ }_{j+1} \mathbf{x}\right)$.

Remark 1 We have fixed an exact expression in order to simplify the proof of Theorem 1 . The reader can check that the generation that is looked upon when the first criterion is not decisive can be chosen in any random manner. More precisely, take any map $\nu:[0,1] \longrightarrow \mathbb{N}$ and define $u_{\nu}: \mathbf{X} \longrightarrow[0,1]^{2}$ according to $u_{\nu}(\mathbf{x})=\left(\liminf _{n}\left(x_{n}\right), x_{\nu\left(\liminf _{n}\left(x_{n}\right)\right)}\right)$. If we now define a binary relation $\succcurlyeq_{\nu}$ on $\mathbf{X}$ by the expression: $\mathbf{x} \succcurlyeq_{\nu} \mathbf{y}$ if and only if $u_{\nu}(\mathbf{x})$ lexicographically beats $u_{\nu}(\mathbf{y})$, then a straightforward modification of the argument proves that $\succcurlyeq_{\nu}$ verifies the thesis of Theorem 1 . In the proof above $\nu$ is constantly 1 .

Proposition 1 below shows that with respect to the statement of Theorem 1, possibility remains when $\mathrm{AN}$ and representability are imposed at the cost of NDF and of relaxing WP to the following property (cf., Asheim et al. [2]):

Axiom RWP (Restricted Weak Pareto). If $\mathbf{x}, \mathbf{y} \in \mathbf{X}, \mathbf{x} \gg \mathbf{y}$ and both $\mathbf{x}$ and $\mathbf{y}$ are eventually constant, then $\mathbf{x} \succ \mathbf{y}$.

Proposition 1 There are representable $S W O$ s on $\boldsymbol{X}=[0,1]^{\mathbb{N}}$ that verify $M$, $R W P, H E F, H P, I B P$, and $A N$.

Proof The relation $x \succcurlyeq y$ if and only if $\liminf _{n}\left(x_{n}\right) \geqslant \liminf _{n}\left(y_{n}\right)$ establishes the fact. Observe that the conditions in the statement imply NDP.

Remark 2 We do not yet know if possibility remains when NDF is added to the list of axioms in Proposition 1, irrespective of representability.

In this regard we must emphasize that Theorem 1 is not directly comparable with the result that WP, WHP, WIBP, and AN are incompatible in the case of finite societies because the anonymity axiom is left aside. 


\section{Impossibility results for semicontinuous relations}

In this Section we are interested in the implications of topological semicontinuity. We first produce various impossibility results for SWRs with noninterference properties on $\mathbf{X}=[0,1]^{n}$, a setting where SP and WD coincide. Afterwards we show that they naturally translate into results on $\mathbf{X}=[0,1]^{\mathbb{N}}$. A cardinal variant of the analysis completes this Section.

\subsection{The case of a finite society}

In this context it is known that AN, SP, and IBP (resp., either HE or HP) characterize the extensions of the leximax (resp., leximin): cf., Mariotti and Veneziani [16, Propositions 2, 3]. Let us recall the definitions of these orderings. For any $x \in \mathbb{R}^{n}$, let $\bar{x}$ denote the permutation of $x$ whose components $\bar{x}_{1}, . ., \bar{x}_{n}$ are ranked in ascending order. The leximin ordering $\succcurlyeq^{L M}$ is defined by: $x \succ^{L M}$ $y$ if and only if either $\bar{x}_{1}>\bar{y}_{1}$ or there exists $l>1$ such that $\bar{x}_{1}=\bar{y}_{1}, \ldots$, $\bar{x}_{l-1}=\bar{y}_{l-1}, \bar{x}_{l}>\bar{y}_{l}$. The leximax ordering $\succcurlyeq^{L X}$ is defined by: $x \succ^{L X} y$ if and only if either $\bar{x}_{n}>\bar{y}_{n}$ or there exists $l<n$ such that $\bar{x}_{n}=\bar{y}_{n}, \ldots, \bar{x}_{l+1}=\bar{y}_{l+1}$, $\bar{x}_{l}>\bar{y}_{l}$.

It is now trivial that AN, SP, HP, and IBP are incompatible properties for a SWO on $\mathbf{X}=[0,1]^{n}$ when $n>1$. Relaxing SP to WP produces incompatibility too (in fact WP, WHP, and WIBP together entail dictatorship by an agent, which violates AN), but dropping either HP or IBP instead produces compatibility. Any dictatorship by an agent proves that the incompatibility among AN, SP, HP, and IBP is avoided if AN is dropped and SP is relaxed to $\mathrm{M}$ plus WP.

Since the extensions of the leximax (resp., leximin) do not verify lower (resp., upper) semicontinuity with respect to the sup topology, trivial impossibility consequences follow. ${ }^{1}$ To be precise: No SWO on $\mathbf{X}=[0,1]^{n}$ verifies AN, SP, HP (res., IBP), and USC (resp., LSC). In this Subsection we clarify the extent of the conflict among non-interference principles, Paretian axioms, and semicontinuity by proving that (a) AN and transitivity play no role in such incompatibilities, and (b) if an extremely mild technical condition replaces WD/SP then we still obtain conflicting axiomatics.

Regarding our first purpose, the following Propositions 2 and 3 are in order:

Proposition 2 There is no complete $S W R \succcurlyeq$ on $\boldsymbol{X}=[0,1]^{n}, n \in\{2,3, \ldots\}$, that verifies WIBP, WD, and $L S C$.

Proof We prove that the combination of properties in the statement conveys an absurd conclusion. Let us first show $(0,1,0, \ldots, 0) \succcurlyeq\left(\frac{1}{2}, 1-\frac{1}{i}, 0, \ldots, 0\right)$ for

\footnotetext{
${ }^{1}$ Consider the case of the leximax. For each $i \in \mathbb{N}$ let $\mathbf{y}^{(i)}=\left(1-\frac{1}{i}, \frac{1}{2}\right)$. With respect to the sup topology, $\mathbf{y}^{(i)}$ converges to $\mathbf{y}=\left(1, \frac{1}{2}\right)$. However $(1,0) \succ^{L X} \mathbf{y}^{(i)}$ and $\mathbf{y} \succ^{L X}(1,0)$. Now consider the case of the leximin. For each $i \in \mathbb{N}$ let $\mathbf{x}^{(i)}=\left(\frac{1}{i}, \frac{1}{2}\right)$. With respect to the sup topology, $\mathbf{x}^{(i)}$ converges to $\mathbf{x}=\left(0, \frac{1}{2}\right)$. However $\mathbf{x}^{(i)} \succ^{L M}(0,1)$ and $(0,1) \succ^{L M} \mathbf{x}$.
} 
each $i=2,3, \ldots$. Suppose the opposite, thus $\left(\frac{1}{2}, 1-\frac{1}{i_{0}}, 0, \ldots, 0\right) \succ(0,1,0, \ldots, 0)$ for some $i_{0} \in\{2,3, \ldots\}$. An appeal to WIBP yields $\left(1,1-\frac{1}{i_{0}}, 0, \ldots, 0\right) \succcurlyeq$ $\left(1-\frac{1}{m}, 1,0, \ldots, 0\right)$ for each $m=2,3, \ldots$ Now LSC entails $\left(1,1-\frac{1}{i_{0}}, 0, \ldots, 0\right) \succcurlyeq$ $(1,1,0, \ldots, 0)$, contradicting WD.

With respect to the sup topology, $\left\{\left(\frac{1}{2}, 1-\frac{1}{i}, 0, \ldots, 0\right)\right\}_{i}$ converges to $\left(\frac{1}{2}, 1,0, \ldots, 0\right)$ thus LSC entails $(0,1,0, \ldots, 0) \succcurlyeq\left(\frac{1}{2}, 1,0, \ldots, 0\right)$, contradicting WD.

Proposition 3 There is no complete $S W R \succcurlyeq$ on $\boldsymbol{X}=[0,1]^{n}, n \in\{2,3, \ldots\}$, that verifies WHP, WD, and USC.

Proof We prove that the combination of properties in the statement conveys an absurd conclusion. Let us first show $\left(\frac{1}{i}, \frac{1}{2}, 0, \ldots, 0\right) \succcurlyeq(0,1,0, \ldots, 0)$ for each $i=2,3, \ldots$. Suppose the opposite, thus $(0,1,0, \ldots, 0) \succ\left(\frac{1}{i_{0}}, \frac{1}{2}, 0, \ldots, 0\right)$ for some $i_{0} \in\{2,3, \ldots\}$. An appeal to WHP yields $\left(0, \frac{1}{m}, 0, \ldots, 0\right) \succcurlyeq\left(\frac{1}{i_{0}}, 0,0, \ldots, 0\right)$ for each $m=2,3, \ldots$ Now USC entails $(0,0,0, \ldots, 0) \succcurlyeq\left(\frac{1}{i_{0}}, 0,0, \ldots, 0\right)$, contradicting WD.

With respect to the sup topology, $\left\{\left(\frac{1}{i}, \frac{1}{2}, 0, \ldots, 0\right)\right\}_{i}$ converges to $\left(0, \frac{1}{2}, 0, \ldots, 0\right)$ thus USC entails $\left(0, \frac{1}{2}, 0, \ldots, 0\right) \succcurlyeq(0,1,0, \ldots, 0)$, contradicting WD.

Regarding objective (b), we preliminarily explore the intimate relationship between the Harm Principle and Hammond Equity. This reveals another conflict between HP and USC, which bears comparison with the conclusion in Proposition 3.

Mariotti and Veneziani [15, Prop. 3] proved that when $\mathbf{X}=\mathbb{R}^{2}, \mathrm{WD} / \mathrm{SP}$ and HE imply HP. These authors also proved that when $\mathbf{X}=[0,1]^{n}, n>1$, $\mathrm{HP}$ and $\mathrm{HE}$ are equivalent in the presence of $\mathrm{AN}$ and WD/SP (cf., [14, p. 127]). In fact Lombardi and Veneziani [12, Theorem 1] prove that even WHP is equivalent to $\mathrm{HE}$ in the same circumstances. Proposition 4 below shows that it is possible to deduce the egalitarian HE from HP if the generations are treated equally. The argument is exported to the case of infinitely-lived societies in subsection 4.2 below.

Proposition 4 (Maniquet) Let $\succcurlyeq$ be a $S W O$ on $\boldsymbol{X}=[0,1]^{n}$ for some $n \in$ $\{2,3, \ldots\}$. If $\succcurlyeq$ verifies $A N$ and $H P$ then $\succcurlyeq$ verifies $H E .{ }^{2}$

Proof Suppose the SWO $\succcurlyeq$ verifies AN and HP but not HE. Rejecting HE in the presence of AN ensures that there exist $x_{2}>y_{2}>y_{1}>x_{1}$ such that $\mathbf{x}=$ $\left(x_{1}, x_{2}, x_{3}, \ldots, x_{n}\right) \succ \mathbf{y}=\left(y_{1}, y_{2}, x_{3}, \ldots, x_{n}\right), \mathbf{x}, \mathbf{y} \in[0,1]^{n}$. By reflexivity and $\mathrm{AN},\left(x_{1}, x_{2}, x_{3}, \ldots, x_{n}\right) \sim\left(x_{2}, x_{1}, x_{3}, \ldots, x_{n}\right)$. By transitivity, $\left(x_{2}, x_{1}, x_{3}, \ldots, x_{n}\right) \succ$ $\left(y_{1}, y_{2}, x_{3}, \ldots, x_{n}\right)$ but now the HP assures $\left(y_{2}, x_{1}, x_{3}, \ldots, x_{n}\right) \succ\left(x_{1}, y_{2}, x_{3}, \ldots, x_{n}\right)$, violating $\mathrm{AN}$.

Proposition 4 permits to derive Corollary 2 below by appealing to our next Proposition, which relies on the following minimal sensitivity condition for binary relations $\succcurlyeq$ on $\mathbf{X}=[0,1]^{n}, n \in\{2,3, \ldots\}$ :

2 This result by F. Maniquet has been communicated to the author by R. Veneziani. 
Axiom S-1 There exists $\mathbf{x} \in \mathbf{X}$ such that $\mathbf{x} \nsucc \mathbf{y}=\left(y_{1}, x_{2}, \ldots, x_{n}\right)$ and $y_{1}>x_{1}>x_{2}$.

Proposition 5 There does not exist any $S W R \succcurlyeq$ on $\boldsymbol{X}=[0,1]^{n}, n \in\{2,3, \ldots$.$\} ,$ satisfying $S-1, H E$, and $R U S C{ }^{3}$

Proof By contradiction. Define $\mathbf{y}^{(k)}$ according to: $y_{i}^{(k)}=x_{i}$ if $i=1,3,4, \ldots, n$, $y_{2}^{(k)}=x_{2}+\frac{1}{k}$. With respect to the sup topology, $\mathbf{y}^{(k)}$ converges to $\mathbf{x}$. For each $k>\frac{1}{x_{1}-x_{2}}$, HE entails $\mathbf{y}^{(k)} \succcurlyeq \mathbf{y}$ because $y_{1}>x_{1}=y_{1}^{(k)}>x_{2}+\frac{1}{k}=y_{2}^{(k)}>x_{2}$. This means $\mathbf{x} \succcurlyeq \mathbf{y}$ due to RUSC, contradicting the assumption.

Corollary 2 There does not exist any $S W O \succcurlyeq$ on $\boldsymbol{X}=[0,1]^{n}, n \in\{2,3, \ldots$.$\} ,$ satisfying $S-1, A N, H P$ and $R U S C$.

Proof By Proposition 4, verifies HE. Now Proposition 5 applies.

Our last result in this regard replicates Corollary 2 in terms of IBP and the following minimal sensitivity condition for orderings $\succcurlyeq$ on $\mathbf{X}=[0,1]^{n}$, $n \in\{2,3, \ldots$.$\} :$

Axiom S-2 There exists $\mathbf{x} \in \mathbf{X}$ such that there are $x_{2}>x_{1}>y_{1}$ with $\mathbf{x} \succ \mathbf{y}=\left(y_{1}, x_{2}, \ldots, x_{n}\right)$.

Proposition 6 There does not exist any $S W O \succcurlyeq$ on $\boldsymbol{X}=[0,1]^{n}, n \in$ $\{2,3, \ldots\}$, satisfying $S$-2, $A N, I B P$, and $R L S C$.

Proof By contradiction. Define $\mathbf{x}^{(k)} \in \mathbf{X}$ according to: $x_{i}^{(k)}=x_{i}$ if $i=$ $1,3,4, \ldots, n, x_{2}^{(k)}=x_{2}-\frac{1}{k}$. With respect to the sup topology, $\mathbf{x}^{(k)}$ converges to $\mathbf{x}$. Thus there is $k_{0}$ such that $\mathbf{x}^{(k)} \succ \mathbf{y}$ when $k>k_{0}$, due to RLSC. Select $m>k_{0}$ such that $x_{2}-\frac{1}{m}>y_{1}$. Therefore $\left(x_{1}, x_{2}-\frac{1}{m}, x_{3}, \ldots, x_{n}\right) \succ$ $\left(y_{1}, x_{2}, x_{3}, \ldots, x_{n}\right)$.

By reflexivity and $\mathrm{AN},\left(x_{2}-\frac{1}{m}, x_{1}, x_{3}, \ldots, x_{n}\right) \sim\left(x_{1}, x_{2}-\frac{1}{m}, x_{3}, \ldots, x_{n}\right)$ therefore $\left(x_{2}-\frac{1}{m}, x_{1}, x_{3}, \ldots, x_{n}\right) \succ\left(y_{1}, x_{2}, x_{3}, \ldots, x_{n}\right)$. Now an appeal to IBP yields $\left(x_{2}, x_{1}, x_{3}, \ldots, x_{n}\right) \succ\left(x_{1}, x_{2}, x_{3}, \ldots, x_{n}\right)$, contradicting AN.

Remark 3 The interested reader can mimick the proof of Proposition 6 in order to give a direct argument for Corollary 2 that circumvents Propositions 4 and 5. And alternatively, it is possible to mimick the proof of Proposition 4 in order to prove that AN and IBP entail a 'dual' of HE. ${ }^{4}$ From such implication, Proposition 6 follows easily too.

\footnotetext{
3 Observe that Axiom S-1 holds under e.g., WD/SP. Also, recall that RUSC and USC (resp., RLSC and LSC) coincide in this setting.

4 Such dual property is sometimes used to characterise the leximax. See, e.g., d'Aspremont [7, pp. 56-57].
} 
We can say a few more words about differences in the analysis under WHP/WIBP. These variations make the axioms more distant from standard invariance axioms, with which there is otherwise a contiguity, and their liberal content is even clearer since they emphasise the negative prescriptions of $\mathrm{HP} / \mathrm{IBP}$. Our conclusions in general depend on the specification of the noninterference axioms. Consider the following example:

Example 1 Define a representable SWO $\succcurlyeq$ on $[0,1]^{n}$ by the utility $u(\mathbf{x})=$ $\operatorname{card}\left\{i: x_{i} \geqslant \frac{1}{2}\right\}$. When $n=2, \succcurlyeq$ satisfies AN, RUSC, WHP, WIBP, S-1 (since $\mathbf{y}=(1,0) \succ\left(\frac{1}{3}, 0\right)=\mathbf{x}$ with $\left.y_{1}>x_{1}>x_{2}=y_{2}\right)$ and $\mathrm{S}-2$ (since $\mathbf{x}=\left(\frac{2}{3}, 1\right) \succ(0,1)=\mathbf{y}$ with $\left.x_{2}=y_{2}>x_{1}>y_{1}\right)$, but not HE (since $\mathbf{x}=$ $\left.(1,0) \succ\left(\frac{1}{3}, \frac{1}{4}\right)=\mathbf{y}\right)$. The same is true when $n \geqslant 2$.

Example 1 proves that the impossibility in Corollary 2 is overturned, and the conclusion of Maniquet's Proposition 4 is no longer valid, when WHP replaces HP. Analogously, we can assure that the impossibility in Proposition 6 is overturned if IBP is relaxed to WIBP by appealing to the SWO defined from the utility $v(\mathbf{x})=\operatorname{card}\left\{i: x_{i}>\frac{1}{2}\right\}$.

\subsection{The case of an infinitely-lived society}

Most of the arguments in the preceding subsection carry forward to the case of infinite sequences of utilities. We proceed to discuss the details.

Lombardi and Veneziani [13, Theorem 5] take advantage of their characterizations of the standard leximin/leximax relations that compare infinite streams, in order to prove that there is no weakly complete SWR that verifies AN, SP, a Strong Preference Continuity axiom, HP, and IBP. Here we complement their analysis by appealing to topological continuity instead.

Firstly we study if semicontinuity imposes restrictions to non-interference in the presence of efficiency. In order to convert Propositions 2 and 3 into statements for infinitely-lived societies, neither the completeness axiom nor WD are needed in full capacity. We just need to refer to RWD and the following axiom:

Axiom MC (Minimal Completeness). ${ }^{5}$ If $\mathbf{x}, \mathbf{y} \in \mathbf{X}$, there is $T>1$ such that $\left({ }_{1} \mathbf{x}_{T}, T+1 \mathbf{y}\right) \neq \mathbf{y} \Rightarrow\left({ }_{1} \mathbf{x}_{T}, T+1 \mathbf{y}\right) \succcurlyeq \mathbf{y}$ or $\mathbf{y} \succcurlyeq\left({ }_{1} \mathbf{x}_{T}, T+1 \mathbf{y}\right)$.

With respect to RWD and its reinforcements, a reduction to the case of Propositions 2 and 3 yields Proposition 7 below:

Proposition 7 There is no reflexive $S W R$ on $\boldsymbol{X}=[0,1]^{\mathbb{N}}$ that verifies $M C$, $R W D, I B P($ res., HP), and $R L S C$ (resp., RUSC).

Proof Suppose $\succcurlyeq$ verifies MC, RWD, IBP (res., HP), and RLSC (resp., RUSC). Define a binary relation $R$ on $[0,1]^{2}$ according to: $(x, y) R\left(x^{\prime}, y^{\prime}\right)$ iff $\left(x, y, 0_{\text {con }}\right) \succcurlyeq$

${ }^{5}$ See Lombardi and Veneziani [12, Section 4.1] for a prior use of this axiom. 
$\left(x^{\prime}, y^{\prime}, 0_{\text {con }}\right)$. It is straightforward to check that it is complete because $\succcurlyeq$ is reflexive and MC, and that it verifies IBP (res., HP), WD, and RLSC (resp., RUSC). This contradicts Proposition 2 (resp., Proposition 3).

As has been said, the formal incompatibility between HP-IBP and efficiency properties implying RWD does not remain under $\mathrm{WP}$, since $\mathbf{W}(\mathbf{x})=x_{i}$ produces a M, WP, HEF (if $i>1$ ), HP, IBP, representable and continuous w.r.t. the sup topology (but dictatorial) evaluation. Dictatorship might be avoided by imposing the equal treatment of the generations. This leads us to the question if non-interference is possible under $\mathrm{AN}$ in the presence of continuity. We proceed to check that the answer is essentially negative: The reader can easily borrow the arguments from Proposition 4 to Proposition 6 in order to produce twin statements for infinitely-lived societies, namely Propositions 8 and 9 below. They appeal to the following adapted versions of axioms S-1 and S-2 for orderings $\succcurlyeq$ on $\mathbf{X}=[0,1]^{\mathbb{N}}$ :

Axiom S-1' There exists $\mathbf{x} \in \mathbf{X}$ eventually constant such that there are $y_{1}>x_{1}>x_{2}$ for which $\mathbf{y}=\left(y_{1},{ }_{2} x\right) \succ \mathbf{x}$.

Axiom S-2' There exists $\mathbf{x} \in \mathbf{X}$ eventually constant such that there are $x_{2}>x_{1}>y_{1}$ for which $\mathbf{x} \succ \mathbf{y}=\left(y_{1},{ }_{2} x\right)$.

Proposition 8 Let $\succcurlyeq$ be a SWO on $\boldsymbol{X}=[0,1]^{\mathbb{N}}$. If $\succcurlyeq$ verifies $A N$ and $H P$ then $\succcurlyeq$ verifies $H E$.

Example 1 inspires our next example, which shows that we can not relax HP to WHP in Proposition 8 even if WIBP is guaranteed too:

Example 2 Consider the SWO $\succcurlyeq$ on $[0,1]^{\mathbb{N}}$ defined by: $\mathbf{x} \succcurlyeq \mathbf{y}$ if and only if either $\operatorname{card}\left\{i: x_{i} \geqslant \frac{1}{2}\right\}$ is infinite or $\left(\operatorname{card}\left\{i: x_{i} \geqslant \frac{1}{2}\right\}\right.$ is finite and $\operatorname{card}\{i$ : $\left.\left.x_{i} \geqslant \frac{1}{2}\right\} \geqslant \operatorname{card}\left\{i: y_{i} \geqslant \frac{1}{2}\right\}\right)$. This SWO verifies AN, WHP, WIBP, S- $1^{\prime}$ (since $\mathbf{y}=\left(1,0_{\text {con }}\right) \succ\left(\frac{1}{3}, 0_{\text {con }}\right)=\mathbf{x}$ with $\left.y_{1}>x_{1}>x_{2}\right)$ and S-2' (since $\mathbf{x}=\left(\frac{2}{3}, 1,0_{\text {con }}\right) \succ\left(0,1,0_{\text {con }}\right)=\mathbf{y}$ with $\left.x_{2}>x_{1}>y_{1}\right)$, but not HE (since $\left.\mathbf{x}=\left(1,0_{\text {con }}\right) \succ\left(\frac{1}{3}, \frac{1}{4}, 0_{\text {con }}\right)=\mathbf{y}\right)$. Note that it does not verify RUSC.

Proposition 9 There is no $S W O \succcurlyeq$ on $\boldsymbol{X}=[0,1]^{\mathbb{N}}$ satisfying either of the following sets of conditions:

(a) $S-1^{\prime}, A N, H P$, and $R U S C$, or

(b) $S$ - ' $, A N, I B P$, and $R L S C$.

With respect to Proposition 7, Proposition 9 brings out an incompatibility under a technical condition (a very mild version of RWD) when AN and further consistency are imposed. Proposition 9 is reexplored in Subsection 4.3 below.

4.3 Revisiting the analysis under a cardinal perspective

In order to explore some routes of escape to the generalized impossibilities that stem from semicontinuity, we now check for possible changes in the analysis 
above when well-beings are universally comparable and cardinally measurable. We consider the following cardinal forms of the non-interference principles whose implications we have inspected thus far:

Axiom IEHP (Individual Equal Harm Principle). Suppose $\mathbf{x}, \mathbf{y} \in \mathbf{X}$ are eventually coincident and $\mathbf{x} \succ \mathbf{y}$. Consider two streams $\mathbf{x}^{\prime}, \mathbf{y}^{\prime}$ such that: for some $i \in \mathbb{N}, j \neq i$ implies $x_{j}^{\prime}=x_{j}$ and $y_{j}^{\prime}=y_{j}$. If $x_{i}^{\prime}=x_{i}-\varepsilon$ and $y_{i}^{\prime}=y_{i}-\varepsilon$ for some $\varepsilon>0$ then $x_{i}^{\prime}>y_{i}^{\prime}$ implies $\mathbf{x}^{\prime} \succ \mathbf{y}^{\prime}$.

This axiom is a direct descendant of the Harm Principle thus it captures a related liberal spirit. A similar defense holds for its counterpart:

Axiom IEBP (Individual Equal Benefit Principle). Antecedent as in IEHP, thesis as follows: If $x_{i}^{\prime}=x_{i}+\varepsilon$ and $y_{i}^{\prime}=y_{i}+\varepsilon$ for some $\varepsilon>0$ then $x_{i}^{\prime}>y_{i}^{\prime}$ implies $\mathbf{x}^{\prime} \succ \mathbf{y}^{\prime}$.

The respective versions for finite-length streams are the same except that the restriction of the conclusion to eventually coincident vectors does not apply.

We do not need to explore the context of a finite society in depth because to the effect of comparing the ordinal and cardinal positions, summing up the components is a WD/SP, AN, IEHP, IEBP, continuous with respect to the sup topology evaluation. In fact Mariotti and Veneziani [16] state a property in line with the conjunction of adapted versions of IEHP and IEBP, namely, Uniform Additive Non-Interference. Then they prove that SWOs that verify SP, AN, and Uniform Additive Non-Interference only deviate from the utilitarian ordering in comparisons between indifferent elements for the utilitarian rule.

Let us therefore focus on infinitely-lived societies.

1) Proposition 10 below proves that if a SWO is AN and IEHP then it verifies the following Weak Pigou-Dalton transfer principle (cf., Hara et al. $[11$, p. 185]).

Axiom WPDT (Weak Pigou-Dalton transfer principle). If $\mathbf{x}, \mathbf{y} \in \mathbf{X}$ are such that there is $\varepsilon>0$ with $x_{j}=y_{j}+\varepsilon>y_{j} \geqslant y_{k}>x_{k}=y_{k}-\varepsilon$ for some $j, k \in \mathbb{N}$, and $x_{t}=y_{t}$ when $j \neq t \neq k$, then $\mathbf{y} \succcurlyeq \mathbf{x}$.

Proposition 10 Let $\succcurlyeq$ be a $S W O$ on $\boldsymbol{X}=[0,1]^{\mathbb{N}}$. If $\succcurlyeq$ verifies $A N$ and IEHP then $\succcurlyeq$ verifies WPDT.

Proof Let $\mathbf{x}, \mathbf{y} \in \mathbf{X}$ be such that there is $\varepsilon>0$ with $x_{j}=y_{j}+\varepsilon>y_{j} \geqslant y_{k}>$ $x_{k}=y_{k}-\varepsilon$ for some $j, k \in \mathbb{N}$, and $x_{t}=y_{t}$ when $j \neq t \neq k$. By contradiction, assume $\mathbf{x} \succ \mathbf{y}$. Due to AN we can fix $j=1, k=2$ thus ${ }_{3} \mathbf{x}={ }_{3} \mathbf{y}$, and we also get $\mathbf{x} \succ\left(y_{2}, y_{1},{ }_{3} \mathbf{x}\right)$.

Consider the vectors $\mathbf{x}^{\prime}=\left(x_{1}-\varepsilon,{ }_{2} \mathbf{x}\right)$ and $\mathbf{y}^{\prime}=\left(y_{2}-\varepsilon, y_{1},{ }_{3} \mathbf{x}\right)$. They are obtained from $\mathbf{x}$ and $\left(y_{2}, y_{1},{ }_{3} \mathbf{x}\right)$ by reducing the endowment of their respective presents by $\varepsilon$. Since $x_{1}>y_{2}$ by assumption, we obtain $x_{1}-\varepsilon>y_{2}-\varepsilon$ thus IEHP yields $\mathbf{x}^{\prime}=\left(x_{1}-\varepsilon,{ }_{2} \mathbf{x}\right)=\left(y_{1},{ }_{2} \mathbf{x}\right) \succ \mathbf{y}^{\prime}=\left(y_{2}-\varepsilon, y_{1},{ }_{3} \mathbf{x}\right)=$ $\left(x_{2}, y_{1},{ }_{3} \mathbf{x}\right) \sim\left(y_{1}, x_{2},{ }_{3} \mathbf{x}\right)$, an absurd. 
If we further add $\mathrm{M}$ to the assumptions of Proposition 10, then we also obtain HEF by Asheim et al. [3, Proposition 3].

2) A possibility result emerges from Proposition 7 by replacing HP/IBP with their cardinal variants above:

Proposition 11 There are representable $S W O$ s on $\boldsymbol{X}=[0,1]^{\mathbb{N}}$ that verify $S P$, IEHP and IEBP, RUSC and RLSC. There are reflexive and transitive $S W R$ s on $\boldsymbol{X}=[0,1]^{\mathbb{N}}$ that verify $S P, A N$, IEHP and IEBP.

Proof This reduces to checking that discounted utilitarianism agrees with both IEHP and IEBP, as well as being SP, RUSC and RLSC, and representable. Besides, the utilitarian overtaking and catching up criteria also satisfy IEHP and IEBP, AN, and SP, the cost of anonymity being completeness. ${ }^{6}$

Thus by contrast with the case of general non-interference, utilitarianism can be reconciled with a cardinal approach to these principles.

3 ) It is less obvious that the conclusion in Proposition 9 does not vary if IEHP replaces HP in case $(a)$, and IEBP replaces IBP in case $(b)$. We prove this fact by showing that for infinitely-lived societies, the equal treatment of all generations is incompatible with cardinal non-interference principles under standard semicontinuity and very mild efficiency. We use the following strengthenings of axioms S- $1^{\prime}$ and $\mathrm{S}-2^{\prime}$, which in fact can be rephrased to resemble more the originals although we believe that the technical effort does not pay off. Observe that S-1" and S-2" are weaker than RWD too.

Axiom S-1" There exist $y_{1}>x_{1}>x_{2}$ for which $\mathbf{y}=\left(y_{1},\left(x_{2}\right)_{\text {con }}\right) \succ \mathbf{x}=$ $\left(x_{1},\left(x_{2}\right)_{\text {con }}\right)$

Axiom S-2 ${ }^{\prime \prime}$ There exist $x_{2}>x_{1}>y_{1}$ for which $\mathbf{x}=\left(x_{1},\left(x_{2}\right)_{\text {con }}\right) \succ \mathbf{y}=$ $\left(y_{1},\left(x_{2}\right)_{\text {con }}\right)$.

Proposition 12 There is no $S W O$ on $\boldsymbol{X}=[0,1]^{\mathbb{N}}$ satisfying either of:

(a) $S-1^{\prime \prime}, A N, I E H P$, and $R U S C$, or

(b) $S$-2', $A N, I E B P$, and $R L S C$.

Proof We prove case (b) by contradiction. Suppose $\succcurlyeq$ is a SWO that verifies AN, IEBP, RLSC, and condition $\left(2^{\prime \prime}\right)$. Thus we have $x_{2}>x_{1}>y_{1}$ such that $\mathbf{x}=\left(x_{1},\left(x_{2}\right)_{\text {con }}\right) \succ \mathbf{y}=\left(y_{1},\left(x_{2}\right)_{\text {con }}\right)$. Let us denote $m=x_{1}-y_{1}$.

We now define the following sequence of streams: for each $n$ sufficiently large,

$$
\mathbf{x}^{(n)}=\left(x_{1}-\frac{m}{n}, \ldots \ldots, x_{n}-\frac{m}{n},{ }_{n+1} \mathbf{x}\right) \in \mathbf{X}
$$

6 In fact Asheim and Tungodden [4, Section 5] prove that a property with a formal similarity to the conjunction of IEHP and IEBP, namely 2-Generation Unit Comparability (or 2UC), permits to characterize the overtaking and catching up criteria. However that invariance property incorporates a behavior that has a strongly utilitarian component and cannot be justified from a liberal perspective alone. 
(one only needs $x_{1}-\frac{m}{n}, x_{2}-\frac{m}{n} \in[0,1]$ ). With respect to the sup topology, $\mathbf{x}^{(n)}$ converges to $\mathbf{x}$. Thus there is $n^{\prime} \in \mathbb{N}$ such that $\mathbf{x}^{(n)} \succ \mathbf{y}$ when $n \geqslant n^{\prime}$, due to RLSC.

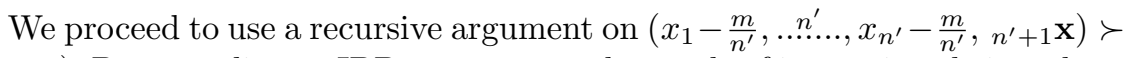
$\left(y_{1},{ }_{2} \mathbf{x}\right)$. By appealing to IBP we compare the result of increasing their endowments to the present by $\frac{m}{n^{\prime}}$ (which utilises $x_{1}>y_{1}+\frac{m}{n^{\prime}}$ or $x_{1}-y_{1}=m>\frac{m}{n^{\prime}}$ ). We then obtain

$$
\left(x_{1}, x_{2}-\frac{m}{n^{\prime}}, \ldots \ldots \ldots . . . ., x_{n^{\prime}}-\frac{m}{n^{\prime}},{ }_{n^{\prime}+1} \mathbf{x}\right) \succ\left(y_{1}+\frac{m}{n^{\prime}},{ }_{2} \mathbf{x}\right)
$$

and due to $\mathrm{AN}$

$$
\left(x_{2}-\frac{m}{n^{\prime}}, x_{1}, x_{3}-\frac{m}{n^{\prime}}, \ldots \ldots \ldots, \ldots, x_{n^{\prime}}-\frac{m}{n^{\prime}},{ }_{n^{\prime}+1} x\right) \succ\left(y_{1}+\frac{m}{n^{\prime}},{ }_{2} x\right)
$$

Again we appeal to IBP in order to compare the result of increasing their endowments to the present by $\frac{m}{n^{\prime}}$ (which now utilises $x_{2}>y_{1}+\frac{2 m}{n^{\prime}}$ or $x_{2}-y_{1}>$ $m \geqslant \frac{2 m}{n^{\prime}}$ because by the recursive assumption $2 \leqslant n^{\prime}$ ). We then obtain

$$
\left(x_{2}, x_{1}, x_{3}-\frac{m}{n^{\prime}}, \ldots \ldots \ldots, \ldots, x_{n^{\prime}}-\frac{m}{n^{\prime}},{ }_{n^{\prime}+1} \mathbf{x}\right) \succ\left(y_{1}+\frac{2 m}{n^{\prime}},{ }_{2} \mathbf{x}\right)
$$

and due to AN

$$
\left(x_{3}-\frac{m}{n^{\prime}}, x_{1}, x_{2}, x_{4}-\frac{m}{n^{\prime}}, \ldots \ldots \ldots, n_{n^{\prime}}-\frac{m}{n^{\prime}},{ }_{n^{\prime}+1} \mathbf{x}\right) \succ\left(y_{1}+\frac{2 m}{n^{\prime}},{ }_{2} \mathbf{x}\right)
$$

Now we repeat the argument until we reach the following conclusion after $n^{\prime}$ steps, which ends the proof:

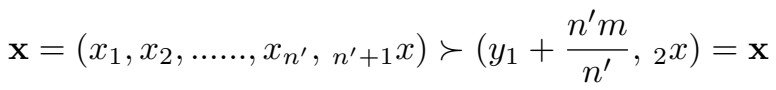

The other instance of the statement is proven by mimicking the proof above.

Remark 4 Continuing our discussion in point 2) above in this Section, Asheim and Tungodden [4, Propositions 4 and 5] in particular prove the existence of reflexive and transitive relations satisfying $\mathrm{SP}, \mathrm{AN}, 2 \mathrm{UC}$ (see footnote 6 ) and two respective forms of preference continuity. As a matter of fact catching up (resp., overtaking) not only verifies SP, AN, 2UC, and Strong (resp., Weak) Preference Continuity, but also IEHP and IEBP. This speaks for the strong restrictions that weak specifications of semicontinuity in the usual sense impose to anonymous and equal harm/benefit behaviors. 


\section{Conclusions and discussion}

We have investigated whether separate non-interference properties (Harm Principle and Individual Benefit Principle, as well as their cardinal variants) are compatible with Paretian relations both in finitely- and infinitely-lived societies. Our analysis adds to prior studies when no further properties are presumed, but we also extend the inspection in order to consider relaxed versions of topological semicontinuities. Our results have the following implications:

1. In the case of finite societies, full non-interference leads to dictatorship when Weak Pareto is guaranteed. We have proved that the situation is quite the opposite when the horizon is infinite (cf., Section 3): Explicit SWOs can be designed that implement enough efficiency (in the form of M plus WP), minimal equity (in the form of HEF), as well as HP plus IBP and non-dictatorships by the present and by the future.

2. If we are interested in imposing standard semicontinuity then we have clarified the extent of the conflict among non-interference principles and Pareto efficiency (cf., Section 4). We proved that renouncing anonymity is not a escape to the incompatibility that arises from the characterizations of the leximin/leximax in the finite context. In the same finite context, if we keep the equal treatment of the generations then a single suitable Paretian comparison yields a conflict. This confirms that for orderings of finite streams, anonymity and $\mathrm{HP} / \mathrm{IBP}$ are almost universally incompatible in the presence of standard semicontinuity. The analysis translates faithfully to infinitely-long streams of utilities. In this case we define cardinal variants of HP/IBP and prove that the conclusion as to keeping anonymity and separate non-interference principles remains negative for semicontinuous SWOs.

3. Asheim and Zuber [6] have introduced continuity axioms that are worth considering because they are compatible with their version of leximin over infinite streams, which is complete and strongly anonymous. Perhaps one could obtain complete leximin or leximax criteria using (restricted versions of) HP or IBP, AN, RWP, and such continuity axioms. But this would be the topic of another paper. ${ }^{7}$

4. We have refrained from introducing the assumption of numerical representability in our analysis. Nevertheless it seems interesting to report on the following related result (cf., Alcantud and García-Sanz [1, Subsection 3.3]): There is no social welfare function on $\mathbf{X}=[0,1]^{\mathbb{N}}$ that verifies RWD and HP (resp., IBP). This is to say, RWD evaluations of the streams must exert some interference (penalising both adverse and favorable changes) on the affairs of particular generations.

\section{Acknowledgements}

I would like to thank the Editor, two anonymous referees, Juan D. MorenoTernero and Roberto Veneziani for their numerous valuable suggestions. Errors

\footnotetext{
7 I thank an anonymous referee for raising this question.
} 
are my own. This work has been supported by the Spanish Ministerio de Ciencia e Innovación under Project ECO2009-07682.

\section{References}

1. J.C.R. Alcantud, M.D. García-Sanz, Evaluations of infinite utility streams: Paretoefficient and egalitarian axiomatics, Mimeo, Universidad de Salamanca.

2. G.B. Asheim, T. Mitra, B. Tungodden, A new equity condition for infinite utility streams and the possibility of being Paretian, in: J. Roemer, K. Suzumura (Eds.), Intergenerational Equity and Sustainability: Conference Proceedings of the IWEA Roundtable Meeting on Intergenerational Equity, Palgrave, 2007.

3. G.B. Asheim, T. Mitra, B. Tungodden, Sustainable recursive social welfare functions, Econ Theory 49 (2012), 267-292.

4. G.B. Asheim, B. Tungodden, Resolving distributional conflicts between generations, Econ Theory 24 (2004), 221-230.

5. G.B. Asheim, B. Tungodden, Do Koopmans' postulates lead to discounted utilitarianism? Discussion Paper 32/04, Norwegian School of Economics and Business Administration.

6. G.B. Asheim, S. Zuber, A complete and strongly anonymous leximin relation on infinite streams. CESifo Working Paper no. 3578 (2011).

7. C. d'Aspremont, Axioms for Social Welfare Orderings, in: L. Hurwicz, D. Schmeidler H. Sonnenschein (Eds.), Social Goals and Social Organization: Essays in Memory of Elisha Pazner, Cambridge University Press, 1985.

8. K. Basu, T. Mitra, Possibility theorems for equitably aggregating infinite utility streams, in: J. Roemer, K. Suzumura (Eds.), Intergenerational Equity and Sustainability: Conference Proceedings of the IWEA Roundtable Meeting on Intergenerational Equity, Palgrave, 2007, pp. 69-84

9. G. Chichilnisky, An axiomatic approach to sustainable development, Soc Choice Welfare 13 (1996), 231-257

10. P. Hammond, Equity, Arrow's conditions, and Rawls' difference principle, Econometrica 44 (1976), 793-804

11. C. Hara, T. Shinotsuka, K. Suzumura, Y. Xu, Continuity and egalitarianism in the evaluation of infinite utility streams, Soc Choice Welfare 31 (2008), 179-191.

12. M. Lombardi, R. Veneziani, Liberal egalitarianism and the Harm Principle, Working Paper No. 649 (2009), Queen Mary, University of London.

13. M. Lombardi, R. Veneziani, Liberal principles for social welfare relations in infinitelylived societies, Working Paper No. 650 (2009), Queen Mary, University of London.

14. M. Mariotti, R. Veneziani, 'Non-interference' implies equality, Soc Choice Welf 32 (2009), 123-128

15. M. Mariotti, R. Veneziani, The paradoxes of the liberal ethics of non-interference, Working Paper No. 653 (2009), Queen Mary, University of London. 
16. M. Mariotti, R. Veneziani, On the impossibility of complete non-interference in Paretian social judgements, Mimeo (2011), Queen Mary, University of London.

17. T. Sakai, Intergenerational preferences and sensitivity to the present, Econ Bulletin 4 (26) (2003), 1-5.

18. T. Sakai, An axiomatic approach to intergenerational equity. Soc Choice Welf 20 (2003), 167-176. 